\title{
Certain new bounds considering the weighted Simpson-like type inequality and applications
}

\author{
Chun-Yan Luo ${ }^{1}$, Ting-Song Du ${ }^{1,2^{*}}$ (D) Mehmet Kunt ${ }^{3}$ and Yao Zhang ${ }^{1}$
}

\section{"Correspondence:}

tingsongdu@ctgu.edu.cn

'Department of Mathematics,

College of Science, China Three

Gorges University, Yichang, China

${ }^{2}$ Three Gorges Mathematical

Research Center, China Three

Gorges University, Yichang, China

Full list of author information is

available at the end of the article

\begin{abstract}
We investigate a weighted Simpson-type identity and obtain new estimation-type results related to the weighted Simpson-like type inequality for the first-order differentiable mappings. We also present some applications to $f$-divergence measures and to higher moments of continuous random variables.
\end{abstract}

MSC: 26A33; 41A55; 26D15; 26E60

Keywords: Simpson's inequality; $(\alpha, m, h)$-convex function; $(\alpha, m, s)$-convex function

\section{Introduction and preliminaries}

The following inequality is named the Simpson integral inequality:

$$
\begin{aligned}
& \left|\frac{1}{6}\left[f\left(r_{1}\right)+4 f\left(\frac{r_{1}+r_{2}}{2}\right)+f\left(r_{2}\right)\right]-\frac{1}{r_{2}-r_{1}} \int_{r_{1}}^{r_{2}} f(x) \mathrm{d} x\right| \\
& \quad \leq \frac{1}{2880}\left\|f^{(4)}\right\|_{\infty}\left(r_{2}-r_{1}\right)^{4},
\end{aligned}
$$

where $f:\left[r_{1}, r_{2}\right] \rightarrow \mathbb{R}$ is a four times continuously differentiable mapping on $\left(r_{1}, r_{2}\right)$, and $\left\|f^{(4)}\right\|_{\infty}=\sup _{t \in\left(r_{1}, r_{2}\right)}\left|f^{(4)}(t)\right|<\infty$.

To see more recent results and the related generalizations with respect to (1.1), we refer the readers to $[1-3,5-18,22-26,29-34]$ and the references therein.

Let us recall that Miheşan [20] presented a class of mappings, called $(\alpha, m)$-convex functions, as follows: A mapping $f:\left[0, b^{*}\right] \rightarrow \mathbb{R}, b^{*}>0$, is said to be $(\alpha, m)$-convex if

$$
f(\lambda x+m(1-\lambda) y) \leq \lambda^{\alpha} f(x)+m\left(1-\lambda^{\alpha}\right) f(y)
$$

for all $x, y \in\left[0, b^{*}\right]$ and $\lambda \in[0,1]$ with some fixed $(\alpha, m) \in(0,1] \times(0,1]$. Shuang et al. [28] proved the following result for such mappings.

Theorem 1.1 Let $f: \mathbb{R}_{0}=[0, \infty) \rightarrow \mathbb{R}$ be a differentiable function on $\mathbb{R}_{0}$, let $r_{1}, r_{2} \in \mathbb{R}_{0}$, $r_{1}<r_{2}$, and let $f^{\prime} \in L^{1}\left[r_{1}, r_{2}\right]$. If $\left|f^{\prime}\right|^{q}$ is $(\alpha, m)$-convex on $\left[0, \frac{r_{2}}{m}\right]$ for $(\alpha, m) \in(0,1] \times(0,1]$ and

(c) The Author(s) 2018. This article is distributed under the terms of the Creative Commons Attribution 4.0 International License (http://creativecommons.org/licenses/by/4.0/), which permits unrestricted use, distribution, and reproduction in any medium, provided you give appropriate credit to the original author(s) and the source, provide a link to the Creative Commons license, and indicate if changes were made. 
$q>1$, then

$$
\begin{aligned}
& \left|\frac{1}{8}\left[f\left(r_{1}\right)+6 f\left(\frac{r_{1}+r_{2}}{2}\right)+f\left(r_{2}\right)\right]-\frac{1}{r_{2}-r_{1}} \int_{r_{1}}^{r_{2}} f(x) \mathrm{d} x\right| \\
& \quad \leq \frac{r_{2}-r_{1}}{4}\left(\frac{(q-1)\left(3^{(2 q-1) /(q-1)}+1\right)}{(2 q-1) 2^{2(2 q-1) /(q-1)}}\right)^{1-\frac{1}{q}} \\
& \quad \times\left\{\left[\frac{1}{1+\alpha}\left|f^{\prime}\left(r_{1}\right)\right|^{q}+\left(\frac{m \alpha}{1+\alpha}\right)\left|f^{\prime}\left(\frac{r_{1}+r_{2}}{2 m}\right)\right|^{q}\right]^{\frac{1}{q}}\right. \\
& \left.+\left[\frac{1}{1+\alpha}\left|f^{\prime}\left(\frac{r_{1}+r_{2}}{2}\right)\right|^{q}+\left(\frac{m \alpha}{1+\alpha}\right)\left|f^{\prime}\left(\frac{r_{2}}{m}\right)\right|^{q}\right]^{\frac{1}{q}}\right\} .
\end{aligned}
$$

Noor et al. [21], introduced the class of $(\alpha, m, h)$-convex functions that unifies several new and known classes of convex functions as follows.

Definition 1.1 ([21]) Let $h: J \subseteq \mathbb{R} \rightarrow \mathbb{R}$. A function $f: I \subseteq \mathbb{R} \rightarrow(0, \infty)$ is said to be $(\alpha, m, h)$-convex function if

$$
f(t x+m(1-t) y) \leq h\left(t^{\alpha}\right) f(x)+m h\left(1-t^{\alpha}\right) f(y)
$$

for all $x, y \in I$ and $t \in[0,1]$ with some fixed $(\alpha, m) \in(0,1] \times(0,1]$.

Note that in [21] the authors have forgotten to write the second $m$ in (1.2) in their original definition.

Let us discuss several particular cases of Definition 1.1.

I. If $h(t)=t^{s}$ for $s \in(0,1]$, then Definition 1.1 reduces to the definition of $(\alpha, m, s)$-convexity.

II. If $h(t)=t^{s}$ for $s \in(0,1]$ and $\alpha=1$, then Definition 1.1 reduces to the definition of $(s, m)$-convexity.

III. If $h(t)=t$, then Definition 1.1 reduces to the definition of $(\alpha, m)$-convexity.

IV. If $h(t)=1$, then Definition 1.1 reduces to the definition of $(m, P)$-convexity.

V. If $h(t)=t(1-t)$ and $\alpha=1$, then Definition 1.1 reduces to the definition of $(m, \operatorname{tgs})$-convexity.

VI. If $h(t)=\frac{\sqrt{1-t}}{2 \sqrt{t}}$ and $\alpha=1$, then Definition 1.1 reduces to the definition of $m-M T$-convexity.

Also, the following theorem was proved in [19]. It obtains an estimation-type result associated with the weighted Simpson-type inequality for $h$-convex mappings using Hölder's inequality.

Theorem 1.2 Let $f:\left[r_{1}, r_{2}\right] \rightarrow \mathbb{R}$ be a differentiable function on $\left(r_{1}, r_{2}\right)$ such that $f^{\prime} \in$ $L^{1}\left[r_{1}, r_{2}\right]$, and let $w:\left[r_{1}, r_{2}\right] \rightarrow \mathbb{R}$ be continuous and symmetric with respect to $\frac{r_{1}+r_{2}}{2}$. If $\left|f^{\prime}\right|^{q}$ 
is $h$-convex on $\left[r_{1}, r_{2}\right]$ for $q>1$ and $p^{-1}+q^{-1}=1$, then

$$
\begin{aligned}
& \left|\frac{1}{6\left(r_{2}-r_{1}\right)}\left[f\left(r_{1}\right)+4 f\left(\frac{r_{1}+r_{2}}{2}\right)+f\left(r_{2}\right)\right] \int_{r_{1}}^{r_{2}} w(x) \mathrm{d} x-\frac{1}{r_{2}-r_{1}} \int_{r_{1}}^{r_{2}} w(x) f(x) \mathrm{d} x\right| \\
& \leq \frac{r_{2}-r_{1}}{12}\|w\|_{\left[r_{1}, r_{2}\right], \infty} \cdot\left(\frac{1+2^{p+1}}{3(p+1)}\right)^{\frac{1}{p}} \cdot 2^{\frac{1}{q}} \\
& \quad \times\left\{\left[\left|f^{\prime}\left(r_{1}\right)\right|^{q} \int_{0}^{\frac{1}{2}} h(t) \mathrm{d} t+\left|f^{\prime}\left(r_{2}\right)\right|^{q} \int_{\frac{1}{2}}^{1} h(t) \mathrm{d} t\right]^{\frac{1}{q}}\right. \\
& \left.\quad+\left[\left|f^{\prime}\left(r_{1}\right)\right|^{q} \int_{\frac{1}{2}}^{1} h(t) \mathrm{d} t+\left|f^{\prime}\left(r_{2}\right)\right|^{q} \int_{0}^{\frac{1}{2}} h(t) \mathrm{d} t\right]^{\frac{1}{q}}\right\} .
\end{aligned}
$$

Different from [19] and [28], our purpose in this paper is to give some new bounds related to the weighted Simpson-like type inequality for the first-order differentiable mappings.

To obtain the principal results, we presume that the absolute value of the derivative of the considered mapping is $(\alpha, m, h)$-convex. Next, we substitute this hypothesis with the boundedness of the derivative and with a Lipschitz condition for the derivative of the considered mapping to establish integral inequalities with new estimation-type results. Also, we provide some applications to $f$-divergence measures and to higher moments of continuous random variables.

\section{Main results}

To obtain our main results, we need the following lemma.

Lemma 2.1 Let $f: I \subseteq \mathbb{R} \rightarrow \mathbb{R}$ be a differentiable function on $I^{\circ}, a, b \in I^{\circ}$ with $a<b$, and let $w:[a, b] \rightarrow \mathbb{R}$ be symmetric with respect to $\frac{a+b}{2}$. If $f^{\prime}, w \in L^{1}[a, b]$, then

$$
\begin{aligned}
& \frac{1}{8(b-a)}\left[f(a)+6 f\left(\frac{a+b}{2}\right)+f(b)\right] \int_{a}^{b} w(x) \mathrm{d} x-\frac{1}{b-a} \int_{a}^{b} w(x) f(x) \mathrm{d} x \\
& =\frac{b-a}{4}\left\{\int_{0}^{1} p_{1}(t) f^{\prime}\left(t a+(1-t) \frac{a+b}{2}\right) \mathrm{d} t\right. \\
& \left.\quad+\int_{0}^{1} p_{2}(t) f^{\prime}\left(t \frac{a+b}{2}+(1-t) b\right) \mathrm{d} t\right\}
\end{aligned}
$$

where

$$
p_{1}(t)=\frac{3}{4} \int_{0}^{1} w\left(s a+(1-s) \frac{a+b}{2}\right) \mathrm{d} s-\int_{0}^{t} w\left(s a+(1-s) \frac{a+b}{2}\right) \mathrm{d} s
$$

and

$$
p_{2}(t)=\frac{1}{4} \int_{0}^{1} w\left(s \frac{a+b}{2}+(1-s) b\right) \mathrm{d} s-\int_{0}^{t} w\left(s \frac{a+b}{2}+(1-s) b\right) \mathrm{d} s
$$


Lu et al. Journal of Inequalities and Applications

(2018) 2018:332

Page 4 of 20

Proof Integrating by parts and changing the variables, we have

$$
\begin{aligned}
\mathcal{I}_{1}= & \int_{0}^{1} p_{1}(t) f^{\prime}\left(t a+(1-t) \frac{a+b}{2}\right) \mathrm{d} t \\
= & \int_{0}^{1}\left[\frac{3}{4} \int_{0}^{1} w\left(s a+(1-s) \frac{a+b}{2}\right) \mathrm{d} s\right. \\
& \left.-\int_{0}^{t} w\left(s a+(1-s) \frac{a+b}{2}\right) \mathrm{d} s\right] f^{\prime}\left(t a+(1-t) \frac{a+b}{2}\right) \mathrm{d} t \\
= & \frac{-2}{b-a}\left[\frac{3}{4} \int_{0}^{1} w\left(s a+(1-s) \frac{a+b}{2}\right) \mathrm{d} s\right. \\
& \left.-\int_{0}^{t} w\left(s a+(1-s) \frac{a+b}{2}\right) \mathrm{d} s\right]\left.f\left(t a+(1-t) \frac{a+b}{2}\right)\right|_{0} ^{1} \\
& -\frac{2}{b-a} \int_{0}^{1} w\left(t a+(1-t) \frac{a+b}{2}\right) f\left(t a+(1-t) \frac{a+b}{2}\right) \mathrm{d} t \\
= & \frac{-2}{b-a}\left[-\frac{1}{4} f(a)-\frac{3}{4} f\left(\frac{a+b}{2}\right)\right] \int_{0}^{1} w\left(s a+(1-s) \frac{a+b}{2}\right) \mathrm{d} s \\
& -\frac{2}{b-a} \int_{0}^{1} w\left(t a+(1-t) \frac{a+b}{2}\right) f\left(t a+(1-t) \frac{a+b}{2}\right) \mathrm{d} t \\
= & \frac{1}{(b-a)^{2}}\left[f(a)+3 f\left(\frac{a+b}{2}\right)\right] \int_{a}^{\frac{a+b}{2}} w(x) \mathrm{d} x-\frac{a}{(b-a)^{2}} \int_{a}^{\frac{a+b}{2}} w(x) f(x) \mathrm{d} x .
\end{aligned}
$$

Similarly, we get

$$
\begin{aligned}
\mathcal{I}_{2}= & \int_{0}^{1} p_{2}(t) f^{\prime}\left(t \frac{a+b}{2}+(1-t) b\right) \mathrm{d} t \\
= & \frac{-2}{b-a}\left[\frac{1}{4} \int_{0}^{1} w\left(s \frac{a+b}{2}+(1-s) b\right) \mathrm{d} s\right. \\
& \left.-\int_{0}^{t} w\left(s \frac{a+b}{2}+(1-s) b\right) \mathrm{d} s\right]\left.f\left(t \frac{a+b}{2}+(1-t) b\right)\right|_{0} ^{1} \\
& -\frac{2}{b-a} \int_{0}^{1} w\left(t \frac{a+b}{2}+(1-t) b\right) f\left(t \frac{a+b}{2}+(1-t) b\right) \mathrm{d} t \\
= & \frac{1}{(b-a)^{2}}\left[3 f\left(\frac{a+b}{2}\right)+f(b)\right] \int_{\frac{a+b}{2}}^{b} w(x) \mathrm{d} x-\frac{4}{(b-a)^{2}} \int_{\frac{a+b}{2}}^{b} w(x) f(x) \mathrm{d} x .
\end{aligned}
$$

Since $w(x)$ is symmetric with respect to $\frac{a+b}{2}$, we have

$$
\int_{a}^{\frac{a+b}{2}} w(x) \mathrm{d} x=\int_{\frac{a+b}{2}}^{b} w(x) \mathrm{d} x=\frac{1}{2} \int_{a}^{b} w(x) \mathrm{d} x .
$$

Thus we have

$$
\begin{aligned}
& \frac{b-a}{4}\left(\mathcal{I}_{1}+\mathcal{I}_{2}\right) \\
& =\frac{1}{8(b-a)}\left[f(a)+6 f\left(\frac{a+b}{2}\right)+f(b)\right] \int_{a}^{b} w(x) \mathrm{d} x-\frac{1}{b-a} \int_{a}^{b} w(x) f(x) \mathrm{d} x,
\end{aligned}
$$

which completes the proof. 
Throughout the work, we write $\|w\|_{[a, b], \infty}=\sup _{x \in[a, b]}|w(x)|$ for a continuous mapping $w:[a, b] \rightarrow \mathbb{R}$. Next, we derive our main results.

Theorem 2.1 Let $f: \mathbb{R}_{0}=[0, \infty) \rightarrow \mathbb{R}$ be a differentiable function on $\mathbb{R}_{0}, a, b \in \mathbb{R}_{0}, a<b$, let $f^{\prime} \in L^{1}[a, b]$, and let $w:[a, b] \rightarrow \mathbb{R}$ be continuous and symmetric with respect to $\frac{a+b}{2}$. If $\left|f^{\prime}\right|^{q}$ for $q \geq 1$ is $(\alpha, m, h)$-convex on $\left[0, \frac{b}{m}\right]$ with some fixed $(\alpha, m) \in(0,1] \times(0,1]$, then

$$
\begin{aligned}
& \left|\frac{1}{8(b-a)}\left[f(a)+6 f\left(\frac{a+b}{2}\right)+f(b)\right] \int_{a}^{b} w(x) \mathrm{d} x-\frac{1}{b-a} \int_{a}^{b} w(x) f(x) \mathrm{d} x\right| \\
& \leq \frac{b-a}{4}\|w\|_{[a, b], \infty}\left(\frac{5}{16}\right){ }^{1-\frac{1}{q}} \\
& \quad \times\left\{\left[\int_{0}^{1}\left|\frac{3}{4}-t\right|\left(h\left(t^{\alpha}\right)\left|f^{\prime}(a)\right|^{q}+h\left(1-t^{\alpha}\right) m\left|f^{\prime}\left(\frac{a+b}{2 m}\right)\right|^{q}\right) \mathrm{d} t\right]^{\frac{1}{q}}\right. \\
& \left.+\left[\int_{0}^{1}\left|\frac{1}{4}-t\right|\left(h\left(t^{\alpha}\right)\left|f^{\prime}\left(\frac{a+b}{2}\right)\right|^{q}+h\left(1-t^{\alpha}\right) m\left|f^{\prime}\left(\frac{b}{m}\right)\right|^{q}\right) \mathrm{d} t\right]^{\frac{1}{q}}\right\} .
\end{aligned}
$$

Proof Applying Lemma 2.1 and using the fact that $\|w\|_{\left[a, \frac{a+b}{2}\right], \infty},\|w\|_{\left[\frac{a+b}{2}, b\right], \infty} \leq\|w\|_{[a, b], \infty}$, we have

$$
\begin{aligned}
&\left|\frac{1}{8(b-a)}\left[f(a)+6 f\left(\frac{a+b}{2}\right)+f(b)\right] \int_{a}^{b} w(x) \mathrm{d} x-\frac{1}{b-a} \int_{a}^{b} w(x) f(x) \mathrm{d} x\right| \\
& \leq \frac{b-a}{4}\left\{\int_{0}^{1} \mid \frac{3}{4} \int_{0}^{1} w\left(s a+(1-s) \frac{a+b}{2}\right) \mathrm{d} s\right. \\
&-\int_{0}^{t} w\left(s a+(1-s) \frac{a+b}{2}\right) \mathrm{d} s\left|f^{\prime}\left(t a+(1-t) \frac{a+b}{2}\right)\right| \mathrm{d} t \\
&+\int_{0}^{1} \mid \frac{1}{4} \int_{0}^{1} w\left(s \frac{a+b}{2}+(1-s) b\right) \mathrm{d} s \\
&\left.-\int_{0}^{t} w\left(s \frac{a+b}{2}+(1-s) b\right) \mathrm{d} s\left|f^{\prime}\left(t \frac{a+b}{2}+(1-t) b\right)\right| \mathrm{d} t\right\} \\
& \leq \frac{b-a}{4}\|w\|_{[a, b], \infty}\left\{\int_{0}^{1}\left|\frac{3}{4} \int_{0}^{1} \mathrm{~d} s-\int_{0}^{t} \mathrm{~d} s\right|\left|f^{\prime}\left(t a+(1-t) \frac{a+b}{2}\right)\right| \mathrm{d} t\right. \\
&\left.+\int_{0}^{1}\left|\frac{1}{4} \int_{0}^{1} \mathrm{~d} s-\int_{0}^{t} \mathrm{~d} s\right| f^{\prime}\left(t \frac{a+b}{2}+(1-t) b\right) \mid \mathrm{d} t\right\} \\
&= \frac{b-a}{4}\|w\|_{[a, b], \infty}\left\{\int_{0}^{1}\left|\frac{3}{4}-t\right|\left|f^{\prime}\left(t a+(1-t) \frac{a+b}{2}\right)\right| \mathrm{d} t\right. \\
&\left.+\int_{0}^{1}\left|\frac{1}{4}-t\right| f^{\prime}\left(t \frac{a+b}{2}+(1-t) b\right) \mid \mathrm{d} t\right\} .
\end{aligned}
$$

Using the power mean inequality, we have

$$
\begin{aligned}
& \left|\frac{1}{8(b-a)}\left[f(a)+6 f\left(\frac{a+b}{2}\right)+f(b)\right] \int_{a}^{b} w(x) \mathrm{d} x-\frac{1}{b-a} \int_{a}^{b} w(x) f(x) \mathrm{d} x\right| \\
& \quad \leq \frac{b-a}{4}\|w\|_{[a, b], \infty}
\end{aligned}
$$




$$
\begin{aligned}
& \times\left\{\left(\int_{0}^{1}\left|\frac{3}{4}-t\right| \mathrm{d} t\right)^{1-\frac{1}{q}}\left[\int_{0}^{1}\left|\frac{3}{4}-t\right|\left|f^{\prime}\left(t a+(1-t) \frac{a+b}{2}\right)\right|^{q} \mathrm{~d} t\right]^{\frac{1}{q}}\right. \\
& \left.+\left(\int_{0}^{1}\left|\frac{1}{4}-t\right| \mathrm{d} t\right)^{1-\frac{1}{q}}\left[\int_{0}^{1}\left|\frac{1}{4}-t\right|\left|f^{\prime}\left(t \frac{a+b}{2}+(1-t) b\right)\right|^{q} \mathrm{~d} t\right]^{\frac{1}{q}}\right\}
\end{aligned}
$$

From (2.3) and (2.4) we get the inquired inequality in (2.2), since

$$
\int_{0}^{1}\left|\frac{1}{4}-t\right| \mathrm{d} t=\int_{0}^{1}\left|\frac{3}{4}-t\right| \mathrm{d} t=\frac{5}{16}
$$

and using the $(\alpha, m, h)$-convexity of $\left|f^{\prime}\right|^{q}$ on $\left[0, \frac{b}{m}\right]$, we have

$$
\left|f^{\prime}\left(t a+(1-t) \frac{a+b}{2}\right)\right|^{q} \leq h\left(t^{\alpha}\right)\left|f^{\prime}(a)\right|^{q}+h\left(1-t^{\alpha}\right) m\left|f^{\prime}\left(\frac{a+b}{2 m}\right)\right|^{q}
$$

and

$$
\left|f^{\prime}\left(t \frac{a+b}{2}+(1-t) b\right)\right|^{q} \leq h\left(t^{\alpha}\right)\left|f^{\prime}\left(\frac{a+b}{2}\right)\right|^{q}+h\left(1-t^{\alpha}\right) m\left|f^{\prime}\left(\frac{b}{m}\right)\right|^{q} .
$$

Direct computation provides the following cases.

Corollary 2.1 If we take $q=1$ in Theorem 2.1, then we have the following inequality for $(\alpha, m, h)$-convex functions:

$$
\begin{aligned}
& \left|\frac{1}{8(b-a)}\left[f(a)+6 f\left(\frac{a+b}{2}\right)+f(b)\right] \int_{a}^{b} w(x) \mathrm{d} x-\frac{1}{b-a} \int_{a}^{b} w(x) f(x) \mathrm{d} x\right| \\
& \leq \frac{b-a}{4}\|w\|_{[a, b], \infty}\left\{\int_{0}^{1}\left|\frac{3}{4}-t\right|\left(h\left(t^{\alpha}\right)\left|f^{\prime}(a)\right|+h\left(1-t^{\alpha}\right) m\left|f^{\prime}\left(\frac{a+b}{2 m}\right)\right|\right) \mathrm{d} t\right. \\
& \left.\quad+\int_{0}^{1}\left|\frac{1}{4}-t\right|\left(h\left(t^{\alpha}\right)\left|f^{\prime}\left(\frac{a+b}{2}\right)\right|+h\left(1-t^{\alpha}\right) m\left|f^{\prime}\left(\frac{b}{m}\right)\right|\right) \mathrm{d} t\right\} .
\end{aligned}
$$

Remark 2.1 Consider Corollary 2.1.

(i) Putting $h(t)=1$, we have the following inequality for $(m, P)$-convex functions:

$$
\begin{aligned}
& \mid \frac{1}{8(b-a)}\left[f(a)+6 f\left(\frac{a+b}{2}\right)+f(b)\right] \int_{a}^{b} w(x) \mathrm{d} x-\frac{1}{b-a} \int_{a}^{b} w(x) f(x) \mathrm{d} x \mid \\
& \leq \frac{5(b-a)}{64}\|w\|_{[a, b], \infty} \\
& \quad \times\left\{\left[\left|f^{\prime}(a)\right|+\left|f^{\prime}\left(\frac{a+b}{2}\right)\right|\right]+m\left[\left|f^{\prime}\left(\frac{a+b}{2 m}\right)\right|+\left|f^{\prime}\left(\frac{b}{m}\right)\right|\right]\right\} .
\end{aligned}
$$


(ii) Putting $h(t)=t(1-t)$ and $\alpha=1$, we have the following inequality for $(m, \operatorname{tgs})$-convex functions:

$$
\begin{aligned}
& \left|\frac{1}{8(b-a)}\left[f(a)+6 f\left(\frac{a+b}{2}\right)+f(b)\right] \int_{a}^{b} w(x) \mathrm{d} x-\frac{1}{b-a} \int_{a}^{b} w(x) f(x) \mathrm{d} x\right| \\
& \leq \frac{71(b-a)}{3 \cdot 2^{11}}\|w\|_{[a, b], \infty}\left\{\left[\left|f^{\prime}(a)\right|+\left|f^{\prime}\left(\frac{a+b}{2}\right)\right|\right]\right. \\
& \left.\quad+m\left[\left|f^{\prime}\left(\frac{a+b}{2 m}\right)\right|+\left|f^{\prime}\left(\frac{b}{m}\right)\right|\right]\right\} .
\end{aligned}
$$

(iii) Putting $h(t)=t^{s}$ and using the inequality $\left(1-t^{\alpha}\right)^{s} \leq 2^{1-s}-t^{s \alpha}$ for $t \in[0,1]$ with some fixed $\alpha \in(0,1], s \in(0,1]$, we have the following inequality for $(\alpha, m, s)$-convex functions:

$$
\begin{aligned}
& \left|\frac{1}{8(b-a)}\left[f(a)+6 f\left(\frac{a+b}{2}\right)+f(b)\right] \int_{a}^{b} w(x) \mathrm{d} x-\frac{1}{b-a} \int_{a}^{b} w(x) f(x) \mathrm{d} x\right| \\
& \leq \frac{b-a}{4}\|w\|_{[a, b], \infty}\left\{\left[\Delta_{1}\left|f^{\prime}(a)\right|+\left(\frac{5 \cdot 2^{1-s}}{16}-\Delta_{1}\right) m\left|f^{\prime}\left(\frac{a+b}{2 m}\right)\right|\right]\right. \\
& \left.+\left[\Delta_{2}\left|f^{\prime}\left(\frac{a+b}{2}\right)\right|+\left(\frac{5 \cdot 2^{1-s}}{16}-\Delta_{2}\right) m\left|f^{\prime}\left(\frac{b}{m}\right)\right|\right]\right\}
\end{aligned}
$$

where

$$
\begin{aligned}
& \Delta_{1}=\frac{3^{s \alpha+2}+2^{2 s \alpha+1} s \alpha-2^{2 s \alpha+2}}{2^{2 s \alpha+3}(s \alpha+1)(s \alpha+2)}, \\
& \Delta_{2}=\frac{1+2^{2 \alpha s+1}(3 \alpha s+2)}{2^{2 \alpha s+3}(s \alpha+1)(s \alpha+2)} .
\end{aligned}
$$

Theorem 2.2 Suppose that all assumptions of Theorem 2.1 are satisfied. Then

$$
\begin{aligned}
& \left|\frac{1}{8(b-a)}\left[f(a)+6 f\left(\frac{a+b}{2}\right)+f(b)\right] \int_{a}^{b} w(x) \mathrm{d} x-\frac{1}{b-a} \int_{a}^{b} w(x) f(x) \mathrm{d} x\right| \\
& \leq \frac{b-a}{4}\|w\|_{[a, b], \infty}\left(\frac{5}{16}\right)^{1-\frac{1}{q}} \\
& \quad \times\left\{\left[\int_{0}^{1}\left(h\left(1-\left(\frac{1-t}{2}\right)^{\alpha}\right) m\left|f^{\prime}\left(\frac{a}{m}\right)\right|^{q}+h\left(\left(\frac{1-t}{2}\right)^{\alpha}\right)\left|f^{\prime}(b)\right|^{q}\right) \mathrm{d} t\right]^{\frac{1}{q}}\right. \\
& \left.+\left[\int_{0}^{1}\left(h\left(\left(\frac{t}{2}\right)^{\alpha}\right)\left|f^{\prime}(a)\right|^{q}+h\left(1-\left(\frac{t}{2}\right)^{\alpha}\right) m\left|f^{\prime}\left(\frac{b}{m}\right)\right|^{q}\right) \mathrm{d} t\right]^{\frac{1}{q}}\right\} .
\end{aligned}
$$

Proof Noting that $t a+(1-t) \frac{a+b}{2}=\frac{1+t}{2} a+\frac{1-t}{2} b$ and using the $(\alpha, m, h)$-convexity of $\left|f^{\prime}\right|^{q}$ on $\left[0, \frac{b}{m}\right]$, for any $t \in[0,1]$, we have the inequality

$$
\begin{aligned}
& \left|f^{\prime}\left(t a+(1-t) \frac{a+b}{2}\right)\right|^{q} \\
& \quad \leq h\left(1-\left(\frac{1-t}{2}\right)^{\alpha}\right) m\left|f^{\prime}\left(\frac{a}{m}\right)\right|^{q}+h\left(\left(\frac{1-t}{2}\right)^{\alpha}\right)\left|f^{\prime}(b)\right|^{q}
\end{aligned}
$$


and, similarly,

$$
\left|f^{\prime}\left(t \frac{a+b}{2}+(1-t) b\right)\right|^{q} \leq h\left(\left(\frac{t}{2}\right)^{\alpha}\right)\left|f^{\prime}(a)\right|^{q}+h\left(1-\left(\frac{t}{2}\right)^{\alpha}\right) m\left|f^{\prime}\left(\frac{b}{m}\right)\right|^{q} .
$$

Continuing from inequality (2.4) in the proof of Theorem 2.1 and using (2.9) and (2.10) with (2.5), we obtain the desired result in (2.8). This completes the proof.

Corollary 2.2 If we take $q=1$ in Theorem 2.2, then the following inequality for $(\alpha, m, h)$ convex functions holds:

$$
\begin{aligned}
& \left|\frac{1}{8(b-a)}\left[f(a)+6 f\left(\frac{a+b}{2}\right)+f(b)\right] \int_{a}^{b} w(x) \mathrm{d} x-\frac{1}{b-a} \int_{a}^{b} w(x) f(x) \mathrm{d} x\right| \\
& \leq \frac{b-a}{4}\|w\|_{[a, b], \infty}\left\{\int_{0}^{1}\left[h\left(1-\left(\frac{1-t}{2}\right)^{\alpha}\right) m\left|f^{\prime}\left(\frac{a}{m}\right)\right|+h\left(\left(\frac{1-t}{2}\right)^{\alpha}\right)\left|f^{\prime}(b)\right|\right] \mathrm{d} t\right. \\
& \left.\quad+\int_{0}^{1}\left[h\left(\left(\frac{t}{2}\right)^{\alpha}\right)\left|f^{\prime}(a)\right|+h\left(1-\left(\frac{t}{2}\right)^{\alpha}\right) m\left|f^{\prime}\left(\frac{b}{m}\right)\right|\right] \mathrm{d} t\right\} .
\end{aligned}
$$

\section{Remark 2.2 Consider Corollary 2.2.}

(i) Putting $h(t)=t^{s}$ for $s \in(0,1]$ and using the inequality $\left(1-t^{\alpha}\right)^{s} \leq 2^{1-s}-t^{s \alpha}$ for $t \in[0,1]$ with some fixed $\alpha \in(0,1]$ and $s \in(0,1]$ again, we have the following inequality for $(\alpha, m, s)$-convex functions:

$$
\begin{aligned}
& \left|\frac{1}{8(b-a)}\left[f(a)+6 f\left(\frac{a+b}{2}\right)+f(b)\right] \int_{a}^{b} w(x) \mathrm{d} x-\frac{1}{b-a} \int_{a}^{b} w(x) f(x) \mathrm{d} x\right| \\
& \leq \frac{b-a}{4}\|w\|_{[a, b], \infty}\left\{\left(2^{1-s}-\left(\frac{2^{-s \alpha}}{1+\alpha s}\right)\right) m\left[\left|f^{\prime}\left(\frac{a}{m}\right)\right|+\left|f^{\prime}\left(\frac{b}{m}\right)\right|\right]\right. \\
& \left.\quad+\left(\frac{2^{-s \alpha}}{1+\alpha s}\right)\left[\left|f^{\prime}(a)\right|+\left|f^{\prime}(b)\right|\right]\right\} .
\end{aligned}
$$

(ii) Putting $h(t)=1$, we have the following inequality for $(m, P)$-convex functions:

$$
\begin{aligned}
& \left|\frac{1}{8(b-a)}\left[f(a)+6 f\left(\frac{a+b}{2}\right)+f(b)\right] \int_{a}^{b} w(x) \mathrm{d} x-\frac{1}{b-a} \int_{a}^{b} w(x) f(x) \mathrm{d} x\right| \\
& \quad \leq \frac{b-a}{4}\|w\|_{[a, b], \infty}\left\{m\left[\left|f^{\prime}\left(\frac{a}{m}\right)\right|+\left|f^{\prime}\left(\frac{b}{m}\right)\right|\right]+\left[\left|f^{\prime}(a)\right|+\left|f^{\prime}(b)\right|\right]\right\} .
\end{aligned}
$$

(iii) Putting $h(t)=t(1-t)$ and $\alpha=1$, we have the following inequality for $(m, \operatorname{tgs})$-convex functions:

$$
\begin{aligned}
& \left|\frac{1}{8(b-a)}\left[f(a)+6 f\left(\frac{a+b}{2}\right)+f(b)\right] \int_{a}^{b} w(x) \mathrm{d} x-\frac{1}{b-a} \int_{a}^{b} w(x) f(x) \mathrm{d} x\right| \\
& \quad \leq \frac{b-a}{24}\|w\|_{[a, b], \infty}\left\{m\left[\left|f^{\prime}\left(\frac{a}{m}\right)\right|+\left|f^{\prime}\left(\frac{b}{m}\right)\right|\right]+\left[\left|f^{\prime}(a)\right|+\left|f^{\prime}(b)\right|\right]\right\} .
\end{aligned}
$$

The next result deals with the case where $\left|f^{\prime}\right|^{q}$ for $q>1$ is $(\alpha, m, h)$-convex. 
Theorem 2.3 Let $f: \mathbb{R}_{0} \rightarrow \mathbb{R}$ be a differentiable function on $\mathbb{R}_{0}, a, b \in \mathbb{R}_{0}, a<b$, let $f^{\prime} \in L^{1}[a, b]$, and let $w:[a, b] \rightarrow \mathbb{R}$ be continuous and symmetric with respect to $\frac{a+b}{2}$. If $\left|f^{\prime}\right|^{q}$ for $q>1$ is $(\alpha, m, h)$-convex on $\left[0, \frac{b}{m}\right]$ with some fixed $(\alpha, m) \in(0,1] \times(0,1]$, then

$$
\begin{gathered}
\left|\frac{1}{8(b-a)}\left[f(a)+6 f\left(\frac{a+b}{2}\right)+f(b)\right] \int_{a}^{b} w(x) \mathrm{d} x-\frac{1}{b-a} \int_{a}^{b} w(x) f(x) \mathrm{d} x\right| \\
\leq \frac{b-a}{4}\|w\|_{[a, b], \infty} \mathcal{Q}^{1-\frac{1}{q}}\left\{\left[\int_{0}^{1}\left(h\left(t^{\alpha}\right)\left|f^{\prime}(a)\right|^{q}+h\left(1-t^{\alpha}\right) m\left|f^{\prime}\left(\frac{a+b}{2 m}\right)\right|^{q}\right) \mathrm{d} t\right]^{\frac{1}{q}}\right. \\
\left.+\left[\int_{0}^{1}\left(h\left(t^{\alpha}\right)\left|f^{\prime}\left(\frac{a+b}{2}\right)\right|^{q}+h\left(1-t^{\alpha}\right) m\left|f^{\prime}\left(\frac{b}{m}\right)\right|^{q}\right) \mathrm{d} t\right]^{\frac{1}{q}}\right\}
\end{gathered}
$$

where

$$
\mathcal{Q}=\frac{(q-1)\left(3^{(2 q-1) /(q-1)}+1\right)}{(2 q-1) 2^{2(2 q-1) /(q-1)}}
$$

Proof Using the Hölder inequality for (2.3), we have

$$
\begin{gathered}
\int_{0}^{1}\left|\frac{3}{4}-t\right|\left|f^{\prime}\left(t a+(1-t) \frac{a+b}{2}\right) \mathrm{d} t\right|+\int_{0}^{1}\left|\frac{1}{4}-t\right|\left|f^{\prime}\left(t \frac{a+b}{2}+(1-t) b\right) \mathrm{d} t\right| \\
\leq\left\{\left(\int_{0}^{1}\left|\frac{3}{4}-t\right|^{\frac{q}{q-1}} \mathrm{~d} t\right)^{1-\frac{1}{q}}\left[\int_{0}^{1}\left|f^{\prime}\left(t a+(1-t) \frac{a+b}{2}\right)\right|^{q} \mathrm{~d} t\right]^{\frac{1}{q}}\right. \\
\left.+\left(\int_{0}^{1}\left|\frac{1}{4}-t\right|^{\frac{q}{q-1}} \mathrm{~d} t\right)^{1-\frac{1}{q}}\left[\int_{0}^{1}\left|f^{\prime}\left(t \frac{a+b}{2}+(1-t) b\right)\right|^{q} \mathrm{~d} t\right]^{\frac{1}{q}}\right\} .
\end{gathered}
$$

From (2.6), (2.7), and (2.12) we get the desired inequality in (2.11), since

$$
\int_{0}^{1}\left|\frac{3}{4}-t\right|^{\frac{q}{q-1}} \mathrm{~d} t=\int_{0}^{1}\left|\frac{1}{4}-t\right|^{\frac{q}{q-1}} \mathrm{~d} t=\frac{(q-1)\left(3^{(2 q-1) /(q-1)}+1\right)}{(2 q-1) 2^{2(2 q-1) /(q-1)}}=\mathcal{Q} .
$$

Now, we state some particular cases of Theorem 2.3.

Corollary 2.3 In Theorem 2.3, putting $h(t)=t^{s}$ and using the inequality $\left(1-t^{\alpha}\right)^{s} \leq 2^{1-s}-$ $t^{s \alpha}$ for $t \in[0,1]$ with some fixed $\alpha \in(0,1], s \in(0,1]$ again, we have the following inequality for $(\alpha, m, s)$-convex functions:

$$
\begin{aligned}
\mid & \frac{1}{8(b-a)}\left[f(a)+6 f\left(\frac{a+b}{2}\right)+f(b)\right] \int_{a}^{b} w(x) \mathrm{d} x-\frac{1}{b-a} \int_{a}^{b} w(x) f(x) \mathrm{d} x \mid \\
\leq & \frac{b-a}{4}\|w\|_{[a, b], \infty} \mathcal{Q}^{1-\frac{1}{q}}\left\{\left[\frac{1}{1+s \alpha}\left|f^{\prime}(a)\right|^{q}+\left(2^{1-s}-\frac{1}{1+s \alpha}\right) m\left|f^{\prime}\left(\frac{a+b}{2 m}\right)\right|^{q}\right]^{\frac{1}{q}}\right. \\
+ & {\left.\left[\frac{1}{1+s \alpha}\left|f^{\prime}\left(\frac{a+b}{2}\right)\right|^{q}+\left(2^{1-s}-\frac{1}{1+s \alpha}\right) m\left|f^{\prime}\left(\frac{b}{m}\right)\right|^{q}\right]^{\frac{1}{q}}\right\} . }
\end{aligned}
$$


Remark 2.3 In Corollary 2.3, if $f(a)=f\left(\frac{a+b}{2}\right)=f(b)$ with $m=1=\alpha$, then the following inequality for $s$-convex functions holds:

$$
\begin{aligned}
\left|f\left(\frac{a+b}{2}\right) \int_{a}^{b} w(x) \mathrm{d} x-\int_{a}^{b} w(x) f(x) \mathrm{d} x\right| \\
\leq \frac{(b-a)^{2}}{4}\|w\|_{[a, b], \infty} \mathcal{Q}^{1-\frac{1}{q}}\left\{\left[\frac{1}{1+s}\left|f^{\prime}(a)\right|^{q}+\left(2^{1-s}-\frac{1}{1+s}\right)\left|f^{\prime}\left(\frac{a+b}{2}\right)\right|^{q}\right]^{\frac{1}{q}}\right. \\
\left.\quad+\left[\frac{1}{1+s}\left|f^{\prime}\left(\frac{a+b}{2}\right)\right|^{q}+\left(2^{1-s}-\frac{1}{1+s}\right)\left|f^{\prime}(b)\right|^{q}\right]^{\frac{1}{q}}\right\} .
\end{aligned}
$$

\section{Corollary 2.4 Consider Theorem 2.3.}

(i) If we take $h(t)=1$, then the following inequality for $(m, P)$-convex functions holds:

$$
\begin{aligned}
& \left|\frac{1}{8(b-a)}\left[f(a)+6 f\left(\frac{a+b}{2}\right)+f(b)\right] \int_{a}^{b} w(x) \mathrm{d} x-\frac{1}{b-a} \int_{a}^{b} w(x) f(x) \mathrm{d} x\right| \\
& \leq \frac{b-a}{4}\|w\|_{[a, b], \infty} \mathcal{Q}^{1-\frac{1}{q}}\left\{\left[\left|f^{\prime}(a)\right|^{q}+m\left|f^{\prime}\left(\frac{a+b}{2 m}\right)\right|^{q}\right]^{\frac{1}{q}}\right. \\
& \left.\quad+\left[\left|f^{\prime}\left(\frac{a+b}{2}\right)\right|^{q}+m\left|f^{\prime}\left(\frac{b}{m}\right)\right|^{q}\right]^{\frac{1}{q}}\right\} .
\end{aligned}
$$

(ii) If we take $h(t)=t(1-t)$ and $\alpha=1$, then the following inequality for ( $m$, tgs)-convex functions holds:

$$
\begin{aligned}
& \left|\frac{1}{8(b-a)}\left[f(a)+6 f\left(\frac{a+b}{2}\right)+f(b)\right] \int_{a}^{b} w(x) \mathrm{d} x-\frac{1}{b-a} \int_{a}^{b} w(x) f(x) \mathrm{d} x\right| \\
& \leq \frac{b-a}{4}\|w\|_{[a, b], \infty} \mathcal{Q}^{1-\frac{1}{q}}\left(\frac{1}{6}\right)^{\frac{1}{q}}\left\{\left[\left|f^{\prime}(a)\right|^{q}+m\left|f^{\prime}\left(\frac{a+b}{2 m}\right)\right|^{q}\right]^{\frac{1}{q}}\right. \\
& \left.\quad+\left[\left|f^{\prime}\left(\frac{a+b}{2}\right)\right|^{q}+m\left|f^{\prime}\left(\frac{b}{m}\right)\right|^{q}\right]^{\frac{1}{q}}\right\} .
\end{aligned}
$$

(iii) If we take $h(t)=\frac{\sqrt{1-t}}{2 \sqrt{t}}$ and $\alpha=1$, then the following inequality for $m-M T$-convex functions holds:

$$
\begin{aligned}
\left|\frac{1}{8(b-a)}\left[f(a)+6 f\left(\frac{a+b}{2}\right)+f(b)\right] \int_{a}^{b} w(x) \mathrm{d} x-\frac{1}{b-a} \int_{a}^{b} w(x) f(x) \mathrm{d} x\right| \\
\leq \frac{b-a}{4}\|w\|_{[a, b], \infty} \mathcal{Q}^{1-\frac{1}{q}}\left(\frac{\pi}{4}\right)^{\frac{1}{q}}\left\{\left[\left|f^{\prime}(a)\right|^{q}+m\left|f^{\prime}\left(\frac{a+b}{2 m}\right)\right|^{q}\right]^{\frac{1}{q}}\right. \\
\left.+\left[\left|f^{\prime}\left(\frac{a+b}{2}\right)\right|^{q}+m\left|f^{\prime}\left(\frac{b}{m}\right)\right|^{q}\right]^{\frac{1}{q}}\right\} .
\end{aligned}
$$

A similar result may be stated. 
Theorem 2.4 Suppose that all assumptions of Theorem 2.3 are satisfied. Then

$$
\begin{aligned}
& \left|\frac{1}{8(b-a)}\left[f(a)+6 f\left(\frac{a+b}{2}\right)+f(b)\right] \int_{a}^{b} w(x) \mathrm{d} x-\frac{1}{b-a} \int_{a}^{b} w(x) f(x) \mathrm{d} x\right| \\
& \leq \frac{b-a}{4}\|w\|_{[a, b], \infty} \mathcal{Q}^{1-\frac{1}{q}} \mid \\
& \quad \times\left\{\left[\int_{0}^{1}\left(h\left(1-\left(\frac{1-t}{2}\right)^{\alpha}\right) m\left|f^{\prime}\left(\frac{a}{m}\right)\right|^{q}+h\left(\left(\frac{1-t}{2}\right)^{\alpha}\right)\left|f^{\prime}(b)\right|^{q}\right) \mathrm{d} t\right]^{\frac{1}{q}}\right. \\
& \left.+\left[\int_{0}^{1}\left(h\left(\left(\frac{t}{2}\right)^{\alpha}\right)\left|f^{\prime}(a)\right|^{q}+h\left(1-\left(\frac{t}{2}\right)^{\alpha}\right) m\left|f^{\prime}\left(\frac{b}{m}\right)\right|^{q}\right) \mathrm{d} t\right]^{\frac{1}{q}}\right\} .
\end{aligned}
$$

Proof The proof of Theorem 2.4 is analogous to that of Theorem 2.3 by using $t a+(1-$ t) $\frac{a+b}{2}=\frac{1+t}{2} a+\frac{1-t}{2} b$ and $\frac{a+b}{2} t+(1-t) b=\frac{t}{2} a+\left(1-\frac{t}{2}\right) b$.

The following result holds for $(\alpha, m, s)$-convexity.

Theorem 2.5 Let $f: \mathbb{R}_{0} \rightarrow \mathbb{R}$ be a differentiable function on $\mathbb{R}_{0}, a, b \in \mathbb{R}_{0}, a<b$, let $f^{\prime} \in$ $L^{1}[a, b]$, and let $w:[a, b] \rightarrow \mathbb{R}$ be continuous and symmetric with respect to $\frac{a+b}{2}$. If $\left|f^{\prime}\right|^{q}$ is $(\alpha, m, s)$-convex on $\left[0, \frac{b}{m}\right]$ for some fixed $(\alpha, m) \in(0,1] \times(0,1], p^{-1}+q^{-1}=1$ and $q>1$, then

$$
\begin{aligned}
& \left|\frac{1}{8(b-a)}\left[f(a)+6 f\left(\frac{a+b}{2}\right)+f(b)\right] \int_{a}^{b} w(x) \mathrm{d} x-\frac{1}{b-a} \int_{a}^{b} w(x) f(x) \mathrm{d} x\right| \\
& \leq \frac{b-a}{4}\|w\|_{[a, b], \infty}\left(\frac{1+3^{p+1}}{(p+1) 4^{p+1}}\right) \\
& \quad \times\left\{\left[\frac{1}{1+s \alpha}\left|f^{\prime}(a)\right|^{q}+\left(2^{1-s}-\frac{1}{1+s \alpha}\right) m\left|f^{\prime}\left(\frac{a+b}{2 m}\right)\right|^{q}\right]^{\frac{1}{q}}\right. \\
& \left.+\left[\frac{1}{1+s \alpha}\left|f^{\prime}\left(\frac{a+b}{2}\right)\right|^{q}+\left(2^{1-s}-\frac{1}{1+s \alpha}\right) m\left|f^{\prime}\left(\frac{b}{m}\right)\right|^{q}\right]^{\frac{1}{q}}\right\} .
\end{aligned}
$$

Proof Since $\left|f^{\prime}\right|^{q}$ is $(\alpha, m, s)$-convex on $\left[0, \frac{b}{m}\right]$, using the Hölder inequality for (2.3), we have

$$
\begin{aligned}
& \left|\frac{1}{8(b-a)}\left[f(a)+6 f\left(\frac{a+b}{2}\right)+f(b)\right] \int_{a}^{b} w(x) \mathrm{d} x-\frac{1}{b-a} \int_{a}^{b} w(x) f(x) \mathrm{d} x\right| \\
& \leq \frac{b-a}{4}\|w\|_{[a, b], \infty}\left\{\left(\int_{0}^{1}\left|\frac{3}{4}-t\right|^{p} \mathrm{~d} t\right)^{\frac{1}{p}}\left[\int_{0}^{1}\left|f^{\prime}\left(t a+(1-t) \frac{a+b}{2}\right)\right|^{q} \mathrm{~d} t\right]^{\frac{1}{q}}\right. \\
& \left.+\left(\left.\int_{0}^{1}\left|\frac{1}{4}-t\right|\right|^{p} \mathrm{~d} t\right)^{\frac{1}{p}}\left[\int_{0}^{1}\left|f^{\prime}\left(t \frac{a+b}{2}+(1-t) b\right)\right|^{q} \mathrm{~d} t\right]^{\frac{1}{q}}\right\} \\
& \leq \frac{b-a}{4}\|w\|_{[a, b], \infty}\left(\frac{1+3^{p+1}}{(p+1) 4^{p+1}}\right)^{\frac{1}{p}} \\
& \quad \times\left\{\left[\left|f^{\prime}(a)\right|^{q} \int_{0}^{1} t^{\alpha s} \mathrm{~d} t+m\left|f^{\prime}\left(\frac{a+b}{2 m}\right)\right|^{q} \int_{0}^{1}\left(1-t^{\alpha}\right)^{s} \mathrm{~d} t\right]^{\frac{1}{q}}\right. \\
& \left.+\left[\left|f^{\prime}\left(\frac{a+b}{2}\right)\right|^{q} \int_{0}^{1} t^{\alpha s} \mathrm{~d} t+m\left|f^{\prime}\left(\frac{b}{m}\right)\right|^{q} \int_{0}^{1}\left(1-t^{\alpha}\right)^{s} \mathrm{~d} t\right]^{\frac{1}{q}}\right\} .
\end{aligned}
$$


From (2.15) we get the desired inequality in (2.14), since

$$
\int_{0}^{1} t^{\alpha s} \mathrm{~d} t=\frac{1}{1+s \alpha}
$$

and using the inequality $\left(1-t^{\alpha}\right)^{s} \leq 2^{1-s}-t^{s \alpha}$ for $t \in[0,1]$ with some fixed $\alpha \in(0,1], s \in$ $[0,1]$, we have

$$
\int_{0}^{1}\left(1-t^{\alpha}\right)^{s} \mathrm{~d} t \leq \int_{0}^{1}\left(2^{1-s}-t^{\alpha s}\right) \mathrm{d} t=2^{1-s}-\frac{1}{1+s \alpha}
$$

Now, we point out a particular case of Theorem 2.5.

Corollary 2.5 If we take $s=1$ and $m=1=\alpha$ in Theorem 2.5, then the following inequality for convex functions holds:

$$
\begin{aligned}
& \left|\frac{1}{8(b-a)}\left[f(a)+6 f\left(\frac{a+b}{2}\right)+f(b)\right] \int_{a}^{b} w(x) \mathrm{d} x-\frac{1}{b-a} \int_{a}^{b} w(x) f(x) \mathrm{d} x\right| \\
& \leq \frac{b-a}{2^{2+\frac{1}{q}}}\|w\|_{[a, b], \infty}\left(\frac{1+3^{p+1}}{(p+1) 4^{p+1}}\right){ }^{\frac{1}{p}}\left\{\left[\left|f^{\prime}(a)\right|^{q}+\left|f^{\prime}\left(\frac{a+b}{2}\right)\right|^{q}\right]^{\frac{1}{q}}\right. \\
& \left.+\left[\left|f^{\prime}\left(\frac{a+b}{2}\right)\right|^{q}+\left|f^{\prime}(b)\right|^{q}\right]^{\frac{1}{q}}\right\} .
\end{aligned}
$$

Next, we would like to point out some published results that are particular cases of the obtained main results.

Remark 2.4 In Lemma 2.1, if we take $w(x)=1$, then identity (2.1) becomes the following equation proved by Shuang et al. [28]:

$$
\begin{aligned}
& \frac{1}{8}\left[f(a)+6 f\left(\frac{a+b}{2}\right)+f(b)\right]-\frac{1}{b-a} \int_{a}^{b} f(x) \mathrm{d} x \\
& \quad=\frac{b-a}{4} \int_{0}^{1}\left[\left(\frac{3}{4}-t\right) f^{\prime}\left(t a+(1-t) \frac{a+b}{2}\right)+\left(\frac{1}{4}-t\right) f^{\prime}\left(t \frac{a+b}{2}+(1-t) b\right)\right] \mathrm{d} t .
\end{aligned}
$$

Remark 2.5 If we take $h(t)=t$ and $w(x)=1$ in Theorems 2.1 and 2.3, then we obtain Theorems 3.1 and 3.5 established by Shuang et al. [28], respectively.

\section{Further estimation results}

If the considered function $f^{\prime}$ is bounded from below and above, then we have the following result.

Theorem 3.1 Let $f: I \subseteq \mathbb{R} \rightarrow \mathbb{R}$ be a differentiable function on $I^{\circ}, a, b \in I^{\circ}, a<b$, and let $w:[a, b] \rightarrow \mathbb{R}$ be continuous and symmetric with respect to $\frac{a+b}{2}$. Assume that $f^{\prime}$ is integrable on $[a, b]$ and there exist constants $m<M$ such that $-\infty<m \leq f^{\prime}(x) \leq M<+\infty$ for all 
Lu et al. Journal of Inequalities and Applications

(2018) 2018:332

Page 13 of 20

$x \in[a, b]$. Then

$$
\begin{aligned}
& \mid \frac{1}{8(b-a)}\left[f(a)+6 f\left(\frac{a+b}{2}\right)+f(b)\right] \int_{a}^{b} w(x) \mathrm{d} x-\frac{1}{b-a} \int_{a}^{b} w(x) f(x) \mathrm{d} x \\
& \quad-\frac{(b-a)(m+M)}{8}\left[\int_{0}^{1} p_{1}(t) \mathrm{d} t+\int_{0}^{1} p_{2}(t) \mathrm{d} t\right] \mid \\
& \quad \leq \frac{5(b-a)(M-m)}{64}\|w\|_{[a, b], \infty},
\end{aligned}
$$

where $p_{1}(t)$ and $p_{2}(t)$ are defined in Lemma 2.1.

Proof From Lemma 2.1 we have

$$
\begin{aligned}
& \frac{1}{8(b-a)}\left[f(a)+6 f\left(\frac{a+b}{2}\right)+f(b)\right] \int_{a}^{b} w(x) \mathrm{d} x-\frac{1}{b-a} \int_{a}^{b} w(x) f(x) \mathrm{d} x \\
& =\frac{b-a}{4}\left\{\int_{0}^{1} p_{1}(t)\left[f^{\prime}\left(t a+(1-t) \frac{a+b}{2}\right)-\frac{m+M}{2}+\frac{m+M}{2}\right] \mathrm{d} t\right. \\
& \left.\quad+\int_{0}^{1} p_{2}(t)\left[f^{\prime}\left(t \frac{a+b}{2}+(1-t) b\right)-\frac{m+M}{2}+\frac{m+M}{2}\right] \mathrm{d} t\right\} \\
& =\frac{b-a}{4}\left\{\int_{0}^{1} p_{1}(t)\left[f^{\prime}\left(t a+(1-t) \frac{a+b}{2}\right)-\frac{m+M}{2}\right] \mathrm{d} t\right. \\
& \left.\quad+\int_{0}^{1} p_{2}(t)\left[f^{\prime}\left(t \frac{a+b}{2}+(1-t) b\right)-\frac{m+M}{2}\right] \mathrm{d} t\right\} \\
& \quad+\frac{(b-a)(m+M)}{8}\left\{\int_{0}^{1} p_{1}(t) \mathrm{d} t+\int_{0}^{1} p_{2}(t) \mathrm{d} t\right\} .
\end{aligned}
$$

So

$$
\begin{aligned}
\mathcal{T}:= & \frac{1}{8(b-a)}\left[f(a)+6 f\left(\frac{a+b}{2}\right)+f(b)\right] \int_{a}^{b} w(x) \mathrm{d} x-\frac{1}{b-a} \int_{a}^{b} w(x) f(x) \mathrm{d} x \\
& -\frac{(b-a)(m+M)}{8}\left[\int_{0}^{1} p_{1}(t) \mathrm{d} t+\int_{0}^{1} p_{2}(t) \mathrm{d} t\right] \\
= & \frac{b-a}{4}\left\{\int_{0}^{1} p_{1}(t)\left[f^{\prime}\left(t a+(1-t) \frac{a+b}{2}\right)-\frac{m+M}{2}\right] \mathrm{d} t\right. \\
& \left.+\int_{0}^{1} p_{2}(t)\left[f^{\prime}\left(t \frac{a+b}{2}+(1-t) b\right)-\frac{m+M}{2}\right] \mathrm{d} t\right\} .
\end{aligned}
$$

Therefore

$$
\begin{aligned}
|\mathcal{T}| \leq & \frac{b-a}{4}\left\{\int_{0}^{1}\left|p_{1}(t)\right| f^{\prime}\left(t a+(1-t) \frac{a+b}{2}\right)-\frac{m+M}{2} \mid \mathrm{d} t\right. \\
& \left.+\int_{0}^{1}\left|p_{2}(t)\right| f^{\prime}\left(t \frac{a+b}{2}+(1-t) b\right)-\frac{m+M}{2} \mid \mathrm{d} t\right\} \\
\leq & \frac{(b-a)(M-m)}{8}\left\{\int_{0}^{1}\left|p_{1}(t)\right| \mathrm{d} t+\int_{0}^{1}\left|p_{2}(t)\right| \mathrm{d} t\right\} .
\end{aligned}
$$


Since $f^{\prime}$ satisfies $-\infty<m \leq f^{\prime}(x) \leq M<+\infty$, we have

$$
m-\frac{m+M}{2} \leq f^{\prime}(x)-\frac{m+M}{2} \leq M-\frac{m+M}{2},
$$

which implies that

$$
\left|f^{\prime}(x)-\frac{m+M}{2}\right| \leq \frac{M-m}{2}
$$

Also, since $w$ is symmetric with respect to $\frac{a+b}{2}$, we get

$$
\begin{aligned}
|\mathcal{T}| \leq & \frac{(b-a)(M-m)}{8} \\
& \times\left\{\int_{0}^{1}\left|\frac{3}{4} \int_{0}^{1} w\left(s a+(1-s) \frac{a+b}{2}\right) \mathrm{d} s-\int_{0}^{t} w\left(s a+(1-s) \frac{a+b}{2}\right) \mathrm{d} s\right| \mathrm{d} t\right. \\
& \left.+\int_{0}^{1}\left|\frac{1}{4} \int_{0}^{1} w\left(s \frac{a+b}{2}+(1-s) b\right) \mathrm{d} s-\int_{0}^{t} w\left(s \frac{a+b}{2}+(1-s) b\right) \mathrm{d} s\right| \mathrm{d} t\right\} \\
\leq & \frac{(b-a)(M-m)}{8}\|w\|_{[a, b], \infty} \\
& \times\left\{\int_{0}^{1}\left|\frac{3}{4} \int_{0}^{1} \mathrm{~d} s-\int_{0}^{t} \mathrm{~d} s\right| \mathrm{d} t+\int_{0}^{1}\left|\frac{1}{4} \int_{0}^{1} \mathrm{~d} s-\int_{0}^{t} \mathrm{~d} s\right| \mathrm{d} t\right\} \\
\leq & \frac{5(b-a)(M-m)}{64}\|w\|_{[a, b], \infty} .
\end{aligned}
$$

This ends the proof.

Corollary 3.1 In Theorem 3.2, if $w(x)=1$, then we get

$$
\left|\frac{1}{8}\left[f(a)+6 f\left(\frac{a+b}{2}\right)+f(b)\right]-\frac{1}{b-a} \int_{a}^{b} f(x) \mathrm{d} x\right| \leq \frac{(b-a)(9 M-m)}{64}
$$

Proof If we take $w(x)=1$, then the relation $\|w\|_{[a, b], \infty}=1$ implies that

$$
\begin{aligned}
& \left|\frac{1}{8}\left[f(a)+6 f\left(\frac{a+b}{2}\right)+f(b)\right]-\frac{1}{b-a} \int_{a}^{b} f(x) \mathrm{d} x\right| \\
& \quad \leq \frac{(b-a)(m+M)}{8}\left|\int_{0}^{1} p_{1}(t) \mathrm{d} t+\int_{0}^{1} p_{2}(t) \mathrm{d} t\right|+\frac{5(b-a)(M-m)}{64} \\
& \quad \leq \frac{(b-a)(m+M)}{8}\left[\left|\int_{0}^{1} p_{1}(t) \mathrm{d} t\right|+\left|\int_{0}^{1} p_{2}(t) \mathrm{d} t\right|\right]+\frac{5(b-a)(M-m)}{64} \\
& \quad \leq \frac{(b-a)(m+M)}{16}+\frac{5(b-a)(M-m)}{64} \\
& \quad=\frac{(b-a)(9 M-m)}{64} .
\end{aligned}
$$

Our next goal is an estimation-type result with respect to the weighted Simpson-like type inequality when the derivative of the considered function $f^{\prime}$ satisfies a Lipschitz condition. 
Theorem 3.2 Let $f: I \subseteq \mathbb{R} \rightarrow \mathbb{R}$ be a differentiable function on $I^{\circ}, a, b \in I^{\circ}, a<b$, and let $w:[a, b] \rightarrow \mathbb{R}$ be continuous and symmetric with respect to $\frac{a+b}{2}$. Assume that $f^{\prime}$ is integrable on $[a, b]$ and satisfies a Lipschitz condition for some $L>0$. Then

$$
\begin{aligned}
& \mid \frac{1}{8(b-a)}\left[f(a)+6 f\left(\frac{a+b}{2}\right)+f(b)\right] \int_{a}^{b} w(x) \mathrm{d} x-\frac{1}{b-a} \int_{a}^{b} w(x) f(x) \mathrm{d} x \\
& \quad-\frac{b-a}{4}\left[f^{\prime}(a) \int_{0}^{1} p_{1}(t) \mathrm{d} t+f^{\prime}(b) \int_{0}^{1} p_{2}(t) \mathrm{d} t\right] \mid \\
& \quad \leq \frac{41(b-a)^{2} L}{3 \cdot 2^{8}}\|w\|_{[a, b], \infty},
\end{aligned}
$$

where $p_{1}(t)$ and $p_{2}(t)$ are defined in Lemma 2.1.

Proof From Lemma 2.1 we have

$$
\begin{aligned}
& \frac{1}{8(b-a)}\left[f(a)+6 f\left(\frac{a+b}{2}\right)+f(b)\right] \int_{a}^{b} w(x) \mathrm{d} x-\frac{1}{b-a} \int_{a}^{b} w(x) f(x) \mathrm{d} x \\
& =\frac{b-a}{4}\left\{\int_{0}^{1} p_{1}(t)\left[f^{\prime}\left(t a+(1-t) \frac{a+b}{2}\right)-f^{\prime}(a)+f^{\prime}(a)\right] \mathrm{d} t\right. \\
& \left.\quad+\int_{0}^{1} p_{2}(t)\left[f^{\prime}\left(t \frac{a+b}{2}+(1-t) b\right)-f^{\prime}(b)+f^{\prime}(b)\right] \mathrm{d} t\right\} \\
& =\frac{b-a}{4}\left\{\int_{0}^{1} p_{1}(t)\left[f^{\prime}\left(t a+(1-t) \frac{a+b}{2}\right)-f^{\prime}(a)\right] \mathrm{d} t\right. \\
& \left.\quad+\int_{0}^{1} p_{2}(t)\left[f^{\prime}\left(t \frac{a+b}{2}+(1-t) b\right)-f^{\prime}(b)\right] \mathrm{d} t\right\} \\
& \quad+\frac{b-a}{4}\left\{\int_{0}^{1} p_{1}(t) f^{\prime}(a) \mathrm{d} t+\int_{0}^{1} p_{2}(t) f^{\prime}(b) \mathrm{d} t\right\} .
\end{aligned}
$$

Then

$$
\begin{aligned}
\mathcal{R}= & \frac{1}{8(b-a)}\left[f(a)+6 f\left(\frac{a+b}{2}\right)+f(b)\right] \int_{a}^{b} w(x) \mathrm{d} x-\frac{1}{b-a} \int_{a}^{b} w(x) f(x) \mathrm{d} x \\
& -\frac{b-a}{4}\left[\int_{0}^{1} p_{1}(t) f^{\prime}(a) \mathrm{d} t+\int_{0}^{1} p_{2}(t) f^{\prime}(b) \mathrm{d} t\right] \\
= & \frac{b-a}{4}\left\{\int_{0}^{1} p_{1}(t)\left[f^{\prime}\left(t a+(1-t) \frac{a+b}{2}\right)-f^{\prime}(a)\right] \mathrm{d} t\right. \\
& \left.+\int_{0}^{1} p_{2}(t)\left[f^{\prime}\left(t \frac{a+b}{2}+(1-t) b\right)-f^{\prime}(b)\right] \mathrm{d} t\right\} .
\end{aligned}
$$

Since $f^{\prime}$ satisfies Lipschitz conditions for some $L>0$, we have

$$
\left|f^{\prime}\left(t a+(1-t) \frac{a+b}{2}\right)-f^{\prime}(a)\right| \leq L\left|t a+(1-t) \frac{a+b}{2}-a\right|=L|1-t|\left(\frac{b-a}{2}\right)
$$

and

$$
\left|f^{\prime}\left(t \frac{a+b}{2}+(1-t) b\right)-f^{\prime}(b)\right| \leq L\left|t \frac{a+b}{2}+(1-t) b-b\right|=L|t|\left(\frac{b-a}{2}\right) .
$$


Hence

$$
|\mathcal{R}| \leq \frac{(b-a)^{2} L}{8}\left\{\int_{0}^{1}(1-t)\left|p_{1}(t)\right| \mathrm{d} t+\int_{0}^{1} t\left|p_{2}(t)\right| \mathrm{d} t\right\} .
$$

Also, since $w$ is symmetric with respect to $\frac{a+b}{2}$, we get

$$
\begin{aligned}
|\mathcal{R}| & \leq \frac{(b-a)^{2} L}{8}\|w\|_{[a, b], \infty}\left\{\int_{0}^{1}(1-t)\left|\frac{3}{4}-t\right| \mathrm{d} t+\int_{0}^{1} t\left|\frac{1}{4}-t\right| \mathrm{d} t\right\} \\
& \leq \frac{41(b-a)^{2} L}{3 \cdot 2^{8}}\|w\|_{[a, b], \infty} .
\end{aligned}
$$

This completes the proof.

Corollary 3.2 In Theorem 3.2, if $w(x)=1$, then we get

$$
\begin{gathered}
\left|\frac{1}{8}\left[f(a)+6 f\left(\frac{a+b}{2}\right)+f(b)\right]-\frac{1}{b-a} \int_{a}^{b} f(x) \mathrm{d} x\right| \\
\leq \frac{41(b-a)^{2} L}{3 \cdot 2^{8}}+\frac{b-a}{16}\left[f^{\prime}(a)+f^{\prime}(b)\right] .
\end{gathered}
$$

\section{Applications}

\section{1 $f$-divergence measures}

In various applications of probability theory, one of the primary themes is discovering a proper measure of distance between any two probability distributions. Let a set $\psi$ and a $\sigma$-finite measure $\mu$ be given and consider the set of all probability densities on $\mu$ defined on

$$
\Omega:=\left\{\rho \mid \rho: \psi \rightarrow \mathbb{R}, \rho(x)>0, \int_{\psi} \rho(x) \mathrm{d} \mu(x)=1\right\} .
$$

Let $f:(0, \infty) \rightarrow \mathbb{R}$ be a given mapping and consider $D_{f}(\rho, \tau)$ defined by

$$
D_{f}(\rho, \tau):=\int_{\psi} \rho(x) f\left[\frac{\tau(x)}{\rho(x)}\right] \mathrm{d} \mu(x), \quad \rho, \tau \in \Omega
$$

If $f$ is convex, then (4.1) is known as the Csisźar $f$-divergence [4].

Shioya and Da-te [27] presented the Hermite-Hadamard $(H H)$ divergence

$$
D_{H H}^{f}(\rho, \tau)=\int_{\psi} \rho(x) \frac{\int_{1}^{\frac{\tau(x)}{\rho(x)}} f(t) \mathrm{d} t}{\frac{\tau(x)}{\rho(x)}-1} \mathrm{~d} \mu(x), \quad \rho, \tau \in \Omega,
$$

where $f$ is convex on $(0, \infty)$ with $f(1)=0$. In the same paper [27], they also gave the property of $H H$ divergence that $D_{H H}^{f}(\rho ; \tau) \geq 0$ with equality if and only if $\rho=\tau$. 
Proposition 4.1 Let all assumptions of Theorem 2.5 hold with $f(1)=0$. If $\rho, \tau \in \Omega$, then

$$
\begin{aligned}
& \left|\frac{1}{8}\left[D_{f}(\rho, \tau)+6 \int_{\psi} \rho(x) f\left(\frac{\rho(x)+\tau(x)}{2 \rho(x)}\right) \mathrm{d} \mu(x)\right]-D_{H H}^{f}(\rho, \tau)\right| \\
& \leq\left(\frac{7}{3 \cdot 2^{9}}\right)^{\frac{1}{2}}\left\{\left[\left|f^{\prime}(1)\right|^{2} \int_{\psi} \frac{(\tau(x)-\rho(x))^{2}}{\rho(x)} \mathrm{d} \mu(x)\right.\right. \\
& \left.\quad+\int_{\psi} \frac{(\tau(x)-\rho(x))^{2}}{\rho(x)}\left|f^{\prime}\left(\frac{\rho(x)+\tau(x)}{2 \rho(x)}\right)\right|^{2} \mathrm{~d} \mu(x)\right]^{\frac{1}{2}} \\
& \quad+\left[\int_{\psi} \frac{(\tau(x)-\rho(x))^{2}}{\rho(x)}\left|f^{\prime}\left(\frac{\rho(x)+\tau(x)}{2 \rho(x)}\right)\right|^{2} \mathrm{~d} \mu(x)\right. \\
& \left.\left.\quad+\int_{\psi} \frac{(\tau(x)-\rho(x))^{2}}{\rho(x)}\left|f^{\prime}\left(\frac{\tau(x)}{\rho(x)}\right)\right|^{2} \mathrm{~d} \mu(x)\right]^{\frac{1}{2}}\right\} .
\end{aligned}
$$

Proof Let $\Psi_{1}=\{x \in \psi: \tau(x)>\rho(x)\}, \Psi_{2}=\{x \in \psi: \tau(x)<\rho(x)\}$, and $\Psi_{3}=\{x \in \psi: \tau(x)=$ $\rho(x)\}$.

Obviously, if $x \in \Psi_{3}$, then equality holds in (4.3). Now if we take $w(x)=1$ and $q=2$ in Corollary 2.5, then for $a=1, b=\frac{\tau(x)}{\rho(x)}$, and $x \in \Psi_{1}$, multiplying both sides of the obtained results by $\rho(x)$ and then integrating on $\Psi_{1}$, we get

$$
\begin{aligned}
& \mid \frac{1}{8}\left[\int_{\Psi_{1}} \rho(x) f\left(\frac{\tau(x)}{\rho(x)}\right) \mathrm{d} \mu(x)+6 \int_{\Psi_{1}} \rho(x) f\left(\frac{\rho(x)+\tau(x)}{2 \rho(x)}\right) \mathrm{d} \mu(x)\right] \\
&-\int_{\Psi_{1}} \rho(x) \frac{\int_{1}^{\frac{\tau(x)}{\rho(x)}} f(t) \mathrm{d} t}{\frac{\tau(x)}{\rho(x)}-1} \mathrm{~d} \mu(x) \mid \\
& \leq\left(\frac{7}{3 \cdot 2^{9}}\right)^{\frac{1}{2}}\left\{\left[\left|f^{\prime}(1)\right|^{2} \int_{\Psi_{1}} \frac{(\tau(x)-\rho(x))^{2}}{\rho(x)} \mathrm{d} \mu(x)\right.\right. \\
&\left.+\int_{\Psi_{1}} \frac{(\tau(x)-\rho(x))^{2}}{\rho(x)}\left|f^{\prime}\left(\frac{\rho(x)+\tau(x)}{2 \rho(x)}\right)\right|^{2} \mathrm{~d} \mu(x)\right]^{\frac{1}{2}} \\
&+\left[\int_{\Psi_{1}} \frac{(\tau(x)-\rho(x))^{2}}{\rho(x)}\left|f^{\prime}\left(\frac{\rho(x)+\tau(x)}{2 \rho(x)}\right)\right|^{2} \mathrm{~d} \mu(x)\right. \\
&\left.\left.+\int_{\Psi_{1}} \frac{(\tau(x)-\rho(x))^{2}}{\rho(x)}\left|f^{\prime}\left(\frac{\tau(x)}{\rho(x)}\right)\right|^{2} \mathrm{~d} \mu(x)\right]^{\frac{1}{2}}\right\} .
\end{aligned}
$$

Similarly, if $x \in \Psi_{2}$, then using Corollary 2.5 for $a=\frac{\tau(x)}{\rho(x)}, b=1$, multiplying both sides of the obtained results by $\rho(x)$, and integrating on $\Psi_{2}$, we get

$$
\begin{aligned}
\mid \frac{1}{8}\left[\int_{\Psi_{2}} \rho(x) f\left(\frac{\tau(x)}{\rho(x)}\right) \mathrm{d} \mu(x)+6 \int_{\Psi_{2}} \rho(x) f\left(\frac{\rho(x)+\tau(x)}{2 \rho(x)}\right) \mathrm{d} \mu(x)\right] \\
-\int_{\Psi_{2}} \rho(x) \frac{\int_{1}^{\frac{\tau(x)}{\rho(x)}} f(t) \mathrm{d} t}{\frac{\tau(x)}{\rho(x)}-1} \mathrm{~d} \mu(x) \mid
\end{aligned}
$$




$$
\begin{aligned}
\leq & \left(\frac{7}{3 \cdot 2^{9}}\right)^{\frac{1}{2}}\left\{\left[\left|f^{\prime}(1)\right|^{2} \int_{\Psi_{2}} \frac{(\tau(x)-\rho(x))^{2}}{\rho(x)} \mathrm{d} \mu(x)\right.\right. \\
& \left.+\int_{\Psi_{2}} \frac{(\tau(x)-\rho(x))^{2}}{\rho(x)}\left|f^{\prime}\left(\frac{\rho(x)+\tau(x)}{2 \rho(x)}\right)\right|^{2} \mathrm{~d} \mu(x)\right]^{\frac{1}{2}} \\
& +\left[\int_{\Psi_{2}} \frac{(\tau(x)-\rho(x))^{2}}{\rho(x)}\left|f^{\prime}\left(\frac{\rho(x)+\tau(x)}{2 \rho(x)}\right)\right|^{2} \mathrm{~d} \mu(x)\right. \\
& \left.\left.+\int_{\Psi_{2}} \frac{(\tau(x)-\rho(x))^{2}}{\rho(x)}\left|f^{\prime}\left(\frac{\tau(x)}{\rho(x)}\right)\right|^{2} \mathrm{~d} \mu(x)\right]^{\frac{1}{2}}\right\} .
\end{aligned}
$$

Adding inequalities (4.4) and (4.5) and then using the triangle inequality, we get the desired result.

\subsection{Random variable}

Suppose that for $0<a<b, w:[a, b] \rightarrow[0,+\infty)$ is a continuous probability density of a continuous random variable $X$ that is symmetric about $\frac{a+b}{2}$. Also, for $r \in \mathbb{R}$, suppose that the $r$ th moment

$$
E_{r}(X)=\int_{a}^{b} x^{r} w(x) \mathrm{d} x
$$

is finite.

Since $w$ is symmetric and $\int_{a}^{b} w(x) \mathrm{d} x=1$, we have

$$
E(X)=\int_{a}^{b} x w(x) \mathrm{d} x=\frac{a+b}{2},
$$

which follows from

$$
\begin{aligned}
\int_{a}^{b} x w(x) \mathrm{d} x & =\int_{a}^{b}(b+a-x) w(b+a-x) \mathrm{d} x \\
& =\int_{a}^{b}(b+a-x) w(x) \mathrm{d} x .
\end{aligned}
$$

Based on the above-mentioned derivations, we obtain the following estimates of the $r$ th moment.

(a) If we consider $f(x)=x^{r}$ on $[a, b]$ for $r \geq 2$, then the function $\left|f^{\prime}(x)\right|^{q}=r^{q} x^{q(r-1)}$ with $q>1$ is a convex function. Therefore, using this function in Remark 2.3 with $s=1$ and in Corollary 2.5, respectively, we have

$$
\begin{aligned}
& \left|(E(X))^{r}-E_{r}(X)\right| \\
& \quad \leq \frac{r(b-a)^{2}}{2^{2+\frac{1}{q}}}\|w\|_{[a, b], \infty} \mathcal{Q}^{1-\frac{1}{q}} \\
& \quad \times\left\{\left[a^{q(r-1)}+\left(\frac{a+b}{2}\right)^{q(r-1)}\right]^{\frac{1}{q}}+\left[\left(\frac{a+b}{2}\right)^{q(r-1)}+b^{q(r-1)}\right]^{\frac{1}{q}}\right\}
\end{aligned}
$$


and

$$
\begin{aligned}
& \left|\frac{a^{r}+b^{r}}{8}+\frac{3}{4}(E(X))^{r}-E_{r}(X)\right| \\
& \leq \frac{r(b-a)^{2}}{2^{2+\frac{1}{q}}}\|w\|_{[a, b], \infty}\left(\frac{1+3^{p+1}}{(p+1) 4^{p+1}}\right)^{\frac{1}{p}} \\
& \quad \times\left\{\left[a^{q(r-1)}+\left(\frac{a+b}{2}\right)^{q(r-1)}\right]^{\frac{1}{q}}+\left[\left(\frac{a+b}{2}\right)^{q(r-1)}+b^{q(r-1)}\right]^{\frac{1}{q}}\right\} .
\end{aligned}
$$

(b) If we consider $f(x)=x^{r}$ on $[a, b]$ for $r \in \mathbb{R}$, then $m=r a^{r-1} \leq f^{\prime}(x)=r x^{r-1} \leq r b^{r-1}=M$, and so from (3.2) in Corollary 3.1 we have

$$
\left|\frac{a^{r}+b^{r}}{8}+\frac{3}{4}(E(X))^{r}-E_{r}(X)\right| \leq \frac{r\left(9 b^{r-1}-a^{r-1}\right)(b-a)}{64} .
$$

(c) If we consider $f(x)=x^{r}$ on $[a, b]$ for $r \in \mathbb{R}$, then the Lipschitz constant $L=\sup _{x \in[a, b]}\left|f^{\prime}(x)\right|=\sup _{x \in[a, b]} r x^{r-1}$ is equivalent to

$$
L= \begin{cases}r b^{r-1}, & r \geq 1 \\ r a^{r-1}, & r<1\end{cases}
$$

So from (3.4) in Corollary 3.2 we have

$$
\left|\frac{a^{r}+b^{r}}{8}+\frac{3}{4}(E(X))^{r}-E_{r}(X)\right|= \begin{cases}\frac{r(b-a)}{16}\left[a^{r-1}+\left(1+\frac{41(b-a)}{48}\right) b^{r-1}\right], & r \geq 1 \\ \frac{r(b-a)}{16}\left[\left(1+\frac{41(b-a)}{48}\right) a^{r-1}+b^{r-1}\right], & r<1\end{cases}
$$

Remark 4.1 Applications based on the obtained results to special means can be given, and we omit the details.

\section{Conclusions}

Based on a new weighted Simpson-like type integral identity, we obtained certain estimation-type results with respect to the weighted Simpson-like type inequality for the first-order differentiable mappings. Some particular cases are considered, which can be derived from the main results in the present paper. It is an interesting topic to apply these estimations to $f$-divergence measures and to higher moments of continuous random variables.

Funding

This work was partially supported by the National Natural Science Foundation of China (No. 61374028) and sponsored by Research Fund for Excellent Dissertation of China Three Gorges University (No. 2018SSPY132 and No. 2018SSPY134).

\section{Competing interests}

The authors declare that they have no competing interests.

\section{Authors' contributions}

All authors contributed equally to writing this paper. All authors read and approved the final manuscript.

\section{Author details}

'Department of Mathematics, College of Science, China Three Gorges University, Yichang, China. ${ }^{2}$ Three Gorges Mathematical Research Center, China Three Gorges University, Yichang, China. ${ }^{3}$ Department of Mathematics, Faculty of Sciences, Karadeniz Technical University, Trabzon, Turkey. 


\section{Publisher's Note}

Springer Nature remains neutral with regard to jurisdictional claims in published maps and institutional affiliations.

\section{Received: 9 October 2018 Accepted: 26 November 2018 Published online: 04 December 2018}

\section{References}

1. Awan, M.U., Noor, K.I., Noor, M.A.: Simpson-type inequalities for geometrically relative convex functions. Ukr. Math. J. 70(7), 992-1000 (2018)

2. Chen, J.H., Huang, X.J.: Some new inequalities of Simpson's type for s-convex functions via fractional integrals. Filomat 31(15), 4989-4997 (2017)

3. Chun, L., Qi, F: Inequalities of Simpson type for functions whose third derivatives are extended s-convex functions and applications to means. J. Comput. Anal. Appl. 19(3), 555-569 (2015)

4. Csisźar, I.: Information-type measures of difference of probability distributions and indirect observations. Studia Sci. Math. Hung. 2, 299-318 (1967)

5. Dragomir, S.S.: Weighted integral inequalities for GG-convex functions. J. Appl. Math. Inform. 36(3), 155-171 (2018)

6. Du, T.S., Li, Y.J., Yang, Z.Q.: A generalization of Simpson's inequality via differentiable mapping using extended $(s, m)$-convex functions. Appl. Math. Comput. 293, 358-369 (2017)

7. Du, T.S., Liao, J.G., Li, Y.J.: Properties and integral inequalities of Hadamard-Simpson type for the generalized $(s, m)$-preinvex functions. J. Nonlinear Sci. Appl. 9, 3112-3126 (2016)

8. Hsu, K.-C., Hwang, S.-R., Tseng, K.-L.: Some extended Simpson-type inequalities and applications. Bull. Iran. Math. Soc. 43(2), 409-425 (2017)

9. Hussain, S., Qaisar, S.: Generalizations of Simpson's type inequalities through preinvexity and prequasiinvexity. Punjab Univ. J. Math. 46(2), 1-9 (2014)

10. Hussain, S., Qaisar, S.: More results on Simpson's type inequality through convexity for twice differentiable continuous mappings. SpringerPlus 5, Article ID 77 (2016)

11. Huy, V.H., Ngô, Q.A.: New inequalities of Simpson-like type involving $n$ knots and the $m$ th derivative. Math. Comput. Model. Dyn. Syst. 52(3-4), 522-528 (2010)

12. Isscan, I.., Turhan, S., Maden, S.: Hermite-Hadamard and Simpson-like type inequalities for differentiable p-quasi-convex functions. Filomat 31(19), 5945-5953 (2017)

13. Kunt, M., İscan, I., Yazici, N., Gözütok, U.: On new inequalities of Hermite-Hadamard-Fejer type for harmonically convex functions via fractional integrals. SpringerPlus 5, Article ID 635 (2016)

14. Latif, M.A., Dragomir, S.S., Momoniat, E.: Some weighted integral inequalities for differentiable $h$-preinvex functions. Georgian Math. J. 25(3), 441-450 (2018)

15. Li, Y.J., Du, T.S.: A generalization of Simpson type inequality via differentiable functions using extended $(s, m)_{\phi}$-preinvex functions. J. Comput. Anal. Appl. 22(4), 613-632 (2017)

16. Li, Y.J., Du, T.S., Yu, B.: Some new integral inequalities of Hadamard-Simpson type for extended $(s, m)$-preinvex functions. Ital. J. Pure Appl. Math. 36, 583-600 (2016)

17. Liu, W.J.: Some Simpson type inequalities for $h$-convex and $(\alpha, m)$-convex functions. J. Comput. Anal. Appl. 16(5), 1005-1012 (2014)

18. Matłoka, M.: Inequalities for $h$-preinvex functions. Appl. Math. Comput. 234, 52-57 (2014)

19. Matłoka, M.: Weighted Simpson type inequalities for $h$-convex functions. J. Nonlinear Sci. Appl. 10, 5770-5780 (2017)

20. Miheşan, V.G.: A generalization of the convexity. Seminar on functional equations. Approx. and Convex, Cluj-Napoca (Romania) (1993)

21. Noor, M.A., Awan, M.U., Noor, K.I., Rassias, T.M.: On ( $\alpha, m, h)$-convexity. Appl. Math. Inf. Sci. 12(1), 145-150 (2018)

22. Pečarić, J., Varošanec, S.: A note on Simpson's inequality for Lipschitzian functions. Soochow J. Math. 27(1), 53-57 (2001)

23. Qaisar, S., He, C.J., Hussain, S.: A generalizations of Simpson's type inequality for differentiable functions using $(\alpha, m)$-convex functions and applications. J. Inequal. Appl. 2013, 158 (2013)

24. Qi, F., Xi, B.Y.: Some integral inequalities of Simpson type for GA- $\varepsilon$-convex functions. Georgian Math. J. 20, 775-788 (2013)

25. Sarikaya, M.Z., Set, E., Özdemir, M.E.: On new inequalities of Simpson's type for s-convex functions. Comput. Math. Appl. 60, 2191-2199 (2010)

26. Set, E., Akdemir, A.O., Özdemir, M.E.: Simpson type integral inequalities for convex functions via Riemann-Liouville integrals. Filomat 31(14), 4415-4420 (2017)

27. Shioya, H., Da-te, T:: A generalisation of Lin divergence and the derivative of a new information divergence. Electron. Commun. Jpn. 78(7), 34-40 (1995)

28. Shuang, Y., Wang, Y., Qi, F.: Integral inequalities of Simpson's type for $(\alpha, m)$-convex functions. J. Nonlinear Sci. Appl. 9, 6364-6370 (2016)

29. Tunç, M., Gov, E., Balgecti, S.: Simpson type quantum integral inequalities for convex functions. Miskolc Math. Notes 19(1), 649-664 (2018)

30. Tunç, M., Yildiz, Ç., Ekinci, A.: On some inequalities of Simpson's type via $h$-convex functions. Hacet. J. Math. Stat. 42(4), 309-317 (2013)

31. Wang, Y., Wang, S.H., Qi, F.: Simpson type integral inequalities in which the power of the absolute value of the first derivative of the integrand is s-preinvex. Facta Univ., Ser. Math. Inform. 28(2), 151-159 (2013)

32. Xi, B.Y., Qi, F.: Integral inequalities of Simpson type for logarithmically convex functions. Adv. Stud. Contemp. Math. (Kyungshang) 23(4), 559-566 (2013)

33. Yang, Z.Q., Li, Y.J., Du, T.S.: A generalization of Simpson type inequality via differentiable functions using $(s, m)$-convex functions. Ital. J. Pure Appl. Math. 35, 327-338 (2015)

34. Zhou, C., Peng, C., Du, T.S.: Simpson-like type inequalities for relative semi- $(\alpha, m)$-logarithmically convex functions. J. Nonlinear Sci. Appl. 10(8), 4485-4498 (2017) 